\title{
Cirurgia no tratamento da síndrome da apneia obstrutiva do sono
}

\author{
Lucas N. A. Lemes ${ }^{1,2 *}$, Luciana B. Godoy ${ }^{1}$
}

\begin{abstract}
Resumo
A síndrome da apneia obstrutiva do sono (SAOS) se caracteriza pela redução ou cessação do fluxo de ar durante o sono, que resulta do estreitamento ou colapso da faringe. Pacientes selecionados podem se beneficiar de procedimentos cirúrgicos que têm como objetivos aumentar o espaço volumétrico da via aérea superior e/ou remover sítios específicos de obstrução. Na literatura médica, o papel da cirurgia no tratamento da SAOS e a eficácia de cada procedimento são controversos. Várias técnicas cirúrgicas propostas ao longo das últimas décadas foram abandonadas, mas muitas outras ainda permanecem potencialmente bem indicadas. Aqui são descritos e comentados os procedimentos mais usados na prática cirúrgica atual para o tratamento da SAOS. É difícil prever o resultado cirúrgico sobre os eventos obstrutivos nestes pacientes, principalmente nos mais graves, mas com indicação criteriosa costuma haver melhora sintomática, ainda que incompleta. Embora a cirurgia possa não ser apropriada para todos os pacientes com SAOS, certos pacientes podem ter benefícios na dependência da finalidade dos procedimentos. Entretanto, mais pesquisas devem ser realizadas quanto à natureza da obstrução em via aérea superior, visando identificar os sítios de obstrução com uma maior padronização das técnicas cirúrgicas para subgrupos específicos. Mais ensaios cirúrgicos randomizados e controlados devem ser feitos no intuito de esclarecer estas questões.
\end{abstract}

Descritores: Transtornos do sono; Apneia do sono tipo obstrutiva; Cirurgia.

\section{Abstract \\ Objective in obstructive sleep apnea syndrome surgical treatment}

Obstructive sleep apnea syndrome (OSA) is characterized by reduction or cessation of airflow during sleep, resulting from the narrowing or collapse of the pharynx. Selected patients may benefit from surgical procedures to increase the volumetric space of the upper airways and/or remove specific sites of obstruction. In the medical literature, the role of surgery in the treatment of OSA and the effectiveness of each procedure are controversial. Several surgical techniques proposed over the past decades have been abandoned, but many others are still potentially well indicated. Here are described and commented the procedures most commonly used in current clinical practice for OSA treatment. It is difficult to predict the surgical outcome of obstructive events in these patients, especially in severe ones, but with careful indication there is usually symptomatic improvement, albeit incomplete. While surgery may not be suitable for all patients with OSA, certain patients may benefit depending on the purpose of the procedures and
1. Serviço de Otorrinolaringologia. Hospital Universitário Pedro Ernesto. Universidade do Estado do Rio de Janeiro. Rio de Janeiro, RJ, Brasil.

2. Disciplina de Otorrinolaringologia. Departamento de Especialidades Cirúrgicas. Faculdade de Ciências Médicas. Rio de Janeiro, RJ, Brasil.

\section{*Endereço para correspondência:}

Serviço de Otorrinolaringologia, HUPE

Boulevard 28 de Setembro, 77, 5ㅇandar

Rio de Janeiro, RJ, Brasil. CEP: 20551-030.

E-mail:drlucaslemes@gmail.com

Revista HUPE, Rio de Janeiro, 2016;15(1):68-74

doi: $10.12957 /$ rhupe.2016.22377

Recebido em 25/02/2016. Aprovado em 22/03/2016.

further research must be made to understand the nature of the obstruction in the upper airways, aiming to identify the obstructive sites with standardizing surgical techniques to specific subgroups. Surgical trials controlled and randomized should continue to clarify these issues.

Keywords: Risk; Cardiovascular diseases; Atherosclerosis; Diabetes mellitus.

\section{Resumen}

Objetivo em tratamento quirúrgico de la apnea obstructiva del sueño

El síndrome de apnea obstructiva del sueño (SAOS) se caracteriza por una reducción o el cese del flujo de aire durante el sueño, como resultado de la reducción o el colapso de la faringe. Los pacientes seleccionados se pueden beneficiar de los procedimientos quirúrgicos que tienen el objetivo de aumentar el espacio volumétrico de la vía respiratoria superior y/o eliminar los sitios específicos de la obstrucción. En la literatura médica, el papel de la cirugía en el tratamiento de SAOS y la eficacia de cada procedimiento son controvertidos. Varias técnicas quirúrgicas utilizadas durante las últimas décadas han sido abandonados, pero muchos otros están siendo potencialmente así se indica. Aquí se describen los procedimientos más comúnmente utilizados en la práctica clínica actual para el tratamiento de la SAOS. Es difícil predecir el resultado quirúrgico de eventos obstructivos en estos pacientes, especialmente en severa, pero con indicación cuidado por lo general hay mejoría de los síntomas, aunque incompleta. Si bien la cirugía puede no ser adecuado para todos los pacientes con SAOS, algunos pacientes se pueden beneficiar dependiendo de la finalidad de los procedimientos 


\section{Artigo de revisão}

y la investigación adicional se debe hacer en cuanto a la naturaleza de la obstrucción de las vías respiratorias superiores, con el objetivo de identificar los sitios de obstrucción con una normalización técnicas quirúrgicas a subgrupos específicos.

\section{Introdução}

A Síndrome da Apneia Obstrutiva do Sono (SAOS) se caracteriza pela redução ou cessação do fluxo de ar durante o sono, que resulta do estreitamento ou colapso da faringe. A síndrome está associada com ronco alto, sono fragmentado e apneias observadas por parceiros de cama ou parentes próximos. Os principais sintomas da SAOS são sonolência diurna e disfunção neurocognitiva, com irritabilidade, alterações de comportamento e humor, dificuldades na concentração e lapsos de memória. ${ }^{1-3}$ Fatores de risco são a obesidade, sexo masculino, retrognatismo, hipotireoidismo, obstrução nasal e ingestão de álcool à noite, com os tratamentos conservadores incluindo perda de peso, modificação da posição do sono do paciente, medicamentos para alívio da obstrução nasal, além de evitar o uso de álcool e hipnóticos. ${ }^{4}$ Apesar de não ser uma nova entidade, o reconhecimento da repercussão clínica da SAOS na área médica só se deu mais recentemente, estando envolvida em problemas relacionados a diversas especialidades como neurologia, pneumologia, otorrinolaringologia,
Ensayos quirúrgicos deben seguir para aclarar estas cuestiones.

Palabras clave: Trastornos del sueño; Apnea del sueño Obstructiva; Cirugía.

cardiologia, geriatria, psiquiatria e pediatria. Além disto, há um papel terapêutico que pode ser desempenhado pela fonoaudiologia, odontologia e fisioterapia, fazendo da SAOS um desafio multidisciplinar tanto do seu diagnóstico quanto no seu tratamento mais eficaz.

O primeiro tratamento cirúrgico preconizado para pacientes com SAOS foi a realização da traqueostomia, evitando assim a passagem do fluxo aéreo respiratório pelo local de obstrução faríngea na via aérea superior (VAS). ${ }^{5}$ Desta forma, o objetivo do traqueostoma é eliminar apneias obstrutivas no sono, podendo ser considerado para este fim um padrão ideal desta eficiência cirúrgica. Porém, a manutenção de uma traqueostomia está associada com morbidade e implicações psicossociais frequentemente inaceitáveis nesta condição. Devido a este inconveniente, a intervenção é raramente utilizada na atualidade, principalmente após ter sido iniciada a partir da década de 1980 a utilização clínica da terapia respiratória por pressão positiva na VAS (CPAP), que apresenta grande eficácia se bem aplicada, mesmo em pacientes com SAOS grave.

Tabela 1. Procedimentos cirúrgicos utilizados na SAOS.

\begin{tabular}{|c|c|c|}
\hline Técnica cirúrgica & Mecanismo de ação & Comentário \\
\hline - Traqueostomia & Transpõe a VAS & $\begin{array}{l}\text { Padrão-ouro pelo acesso cervical direto à via } \\
\text { aérea inferior }\end{array}$ \\
\hline $\begin{array}{l}\text { - Amigdalectomia } \\
\text { - Adenoidectomia }\end{array}$ & Remoção de sítios específicos obstrutivos & Tratamento mais aceito para a SAOS na infância \\
\hline - Epiglotoplastia & $\begin{array}{l}\text { Corrige uma alteração anatômica da } \\
\text { laringe }\end{array}$ & Casos específicos de laringomalácia \\
\hline - Cirurgias nasais & Remoção de sítios obstrutivos nasais & Facilita a adaptação ao CPAP nasal \\
\hline - UPFP tradicional ou a laser & Aumenta o espaço retropalatal & $\begin{array}{l}\text { Ressecção da borda livre de úvula e palato mole, } \\
\text { combinada ou não à amigdalectomia }\end{array}$ \\
\hline - Faringoplastia lateral & $\begin{array}{l}\text { Aumenta o espaço parafaríngeo } \\
\text { e retropalatal }\end{array}$ & $\begin{array}{l}\text { Associa uma UPFP à ressecção muscular constric- } \\
\text { tora na VAS }\end{array}$ \\
\hline $\begin{array}{l}\text { - Glossectomia mediana a laser } \\
\text { e/ou lingualplastia }\end{array}$ & Aumentam o espaço retrolingual & $\begin{array}{l}\text { Técnica promissora aplicada na atualidade com } \\
\text { cirurgia robótica }\end{array}$ \\
\hline $\begin{array}{l}\text { - Osteotomia com avanço max- } \\
\text { ilomandibular }\end{array}$ & $\begin{array}{l}\text { Aumenta o espaço retrolingual } \\
\text { e retropalatal }\end{array}$ & $\begin{array}{l}\text { Maior sucesso estatístico das cirurgias realizadas } \\
\text { na SAOS, mas com alguma morbidade e queixas } \\
\text { associadas }\end{array}$ \\
\hline
\end{tabular}

SAOS: síndrome da apneia obstrutiva do sono; VAS: via aérea superior; UPFP: uvulopalatofaringoplastia. 


\section{Objetivo terapêutico dos procedimentos cirúrgicos}

Pacientes, quando bem selecionados, também podem se beneficiar de outros procedimentos cirúrgicos além da traqueostomia que, sendo realizados com critério, têm como objetivos aumentar o espaço volumétrico das VAS e/ou remover sítios específicos de obstrução (Tabela 1). ${ }^{6}$ É importante salientar que esses procedimentos, ao contrário da traqueostomia, nem sempre conseguem eliminar todos os eventos respiratórios obstrutivos, mas, isoladamente ou quando associados a um tratamento conservador, em muitos casos, conseguem minimizar a sonolência excessiva diurna e outros marcadores clínicos da gravidade da SAOS. ${ }^{2}$ De uma forma geral, em adultos, independentemente de uma adequada seleção dos pacientes, ao longo das últimas décadas as técnicas cirúrgicas que visavam apenas realizar a exérese de tecidos moles supostamente em excesso foram menos eficazes do que as que tiveram como objetivo uma melhora da função neuromuscular na VAS, com reposicionamento de tecidos moles e da estrutura muscular parafaríngea. Várias técnicas propostas foram abandonadas pela evolução do conhecimento nesta área cirúrgica, mas muitas outras ainda permanecem potencialmente com uma boa indicação e resultados clínicos positivos.

No seu atual entendimento fisiopatológico, a apneia obstrutiva do sono se caracteriza como uma disfunção neuromuscular dinâmica no espaço aéreo por onde transita a corrente respiratória, que se apresenta mais estreitado pela hipertrofia muscular concêntrica dos músculos constrictores e/ou dilatadores da faringe. Esse entendimento não era o mesmo dos colegas cirurgiões em um passado recente, que até a década de 1990 atuavam cirurgicamente visando eliminar uma obstrução por tecidos moles em excesso na região da orofaringe, que, de fato, somente na infância é o fator causal típico da SAOS. Assim se compreende o porquê de uma visão contrária à cirurgia muitas vezes manifestada pelo médico clínico que analisa estudos clínicos e revisões da literatura científica da época. ${ }^{7}$ Quando comparados aos efeitos da cirurgia, o adequado uso do CPAP nasal que começou a ser difusamente aplicado realmente mostrou resultados terapêuticos clínicos não intervencionistas bastante satisfatórios. Dada a comprovada eficácia do CPAP nasal, como regra geral, a cirurgia não deve ser recomendada à frente da tentativa inicial deste tratamento. Mas os processos terapêuticos não são excludentes e não há nada que impeça uma associação de medidas clínicas e cirúrgicas na busca do mais adequado controle dos eventos respiratórios obstrutivos anormais.

\section{SAOS na infância}

A SAOS na infância acomete de 7 a 9\% das crianças entre 1 a 10 anos de idade, com maior incidência em pré-escolares de 3 a 6 anos, quando a hipertrofia das tonsilas palatinas e faríngea é mais evidente. ${ }^{8-11} \mathrm{O}$ tratamento cirúrgico em crianças é o mais utilizado e muito melhor estudado em relação aos adultos, com a adenoamigdalectomia levando a resolução do quadro clínico em torno de $80 \%$ dos menores operados.12-16 Já a adenoidectomia, isoladamente, sem exérese de tonsilas palatinas, não costuma ser tão eficaz. ${ }^{12,13} \mathrm{~A}$ adenoamigalectomia, além de melhorar queixas de roncos, também normaliza o crescimento pôndero-estatural, trata a enurese e pode melhorar o desempenho escolar e a função cognitiva da criança, que pode estar sendo afetada pela SAOS. ${ }^{17}$

\section{SAOS em adultos}

Em adultos, o papel da cirurgia no tratamento da SAOS e a eficácia de cada procedimento são mais controversos. A maioria dos estudos que recomendavam cirurgias se basearam em série de casos e estudos observacionais não controlados, com menor significado na literatura científica que aqueles usando CPAP. ${ }^{18-20}$ Estes resultados não recomendam o uso generalizado da intervenção cirúrgica isoladamente como o melhor meio de beneficiar a qualidade do sono em relação a outras opções disponíveis, mas esta resposta tende a variar para cada paciente. Pela análise precisa dos níveis principais de obstrução potencial, mais de um nível pode ser tratado, de forma simultânea ou sequencial, na maior parte das vezes nariz, palato mole e base da língua e/ou hipofaringe. Cirurgias múltiplas abordando vários níveis da VAS têm melhor eficácia como tratamento da SAOS do que as que abordam um único sítio obstrutivo. ${ }^{21}$ Estes procedimentos podem ser um fator auxiliar importante para um melhor uso de outras ferramentas clínicas empregadas no arsenal de opções terapêuticas da SAOS. Assim, é fundamental uma avaliação precisa dos objetivos a serem atingidos e dos seus resultados potenciais para cada procedimento cirúrgico.

\section{O papel do nariz na SAOS}

A obstrução nasal é um dos mecanismos fisiopatogênicos primários de sobrecarga sobre os músculos dilatadores da faringe que favorece o surgimento da 
SAOS. Além dos tratamentos clínicos, com corticosteroides nasais tópicos, a obstrução nasal pode ser corrigida cirurgicamente de forma habitual por septoplastia nasal, ressecções de pólipos e processos inflamatórios endonasais e/ou redução de cornetos nasais hipertróficos. Entretanto, estas cirurgias só melhoram de forma definitiva os eventos respiratórios obstrutivos em uma minoria dos pacientes adultos com a SAOS já instalada. Isso porque a disfunção neuromuscular presente na SAOS ocorre de forma progressiva e ao longo de muitos anos, fazendo com que se estabeleçam alterações anatômicas e funcionais na faringe que são pouco reversíveis a partir de um determinado momento. A melhora da ventilação nasal em um paciente com SAOS intensa é considerada, quando isolada de outros procedimentos, uma terapia adjuvante neste tratamento, sem maior resultado positivo na redução do índice de apneia/ hipopneia (IAH). ${ }^{22}$

Deve-se considerar que a via nasal é a preferencial na aplicação terapêutica do CPAP, sendo mais difícil uma aceitação pelo paciente em termos de conforto terapêutico quando se usa uma máscara oronasal aplicando a pressão simultâneamente em nariz e boca. Também o CPAP pode não ser tão eficiente se houver uma obstrução nasal resistindo à aplicação dessa pressão positiva na VAS, dificultando, assim, seu efeito pneumático sobre os tecidos moles faríngeos, o princípio físico dessa terapia.Já foram realizados estudos em pacientes com SAOS demonstrando uma maior facilidade na adaptação ao CPAP após o tratamento da obstrução nasal. ${ }^{23,24}$

\section{Cirurgias na orofaringe}

A uvulopalatofaringoplastia (UPFP) aumenta a área retropalatal da VAS por ressecção da borda livre da úvula e palato mole e costuma ser combinada com a tonsilectomia das palatinas para um tratamento mais eficaz da SAOS. Esta ressecção do véu palatino tem uma taxa de sucesso global de $40 \%$ em pacientes não selecionados, sendo ainda menos bem sucedida em pacientes obesos. ${ }^{7}$ A maioria dos cirurgiões considera sucesso quando a cirurgia obtém uma redução pela metade do IAH no pós-operatório. Essa taxa de sucesso pode ser de $80 \%$ quando os pacientes são selecionados adequadamente. Essa seleção, por sua vez, depende de um maior grau de hipertrofia das tonsilas palatinas associada a um índice de Mallampati baixo, que é a relação entre a altura de base da língua e a região orofaríngea (Figura 1). ${ }^{6}$ Idealmente não deve haver má oclusão dentária (micro ou retrognatia) e/ou obesidade com IMC (índice de massa corporal) $>30 \mathrm{~kg} / \mathrm{m}^{2}$. As alterações mais comuns no período pós-operatório incluem dor até duas semanas, refluxo nasal com alterações de fala anasalada devido à incompetência palatal transitória,

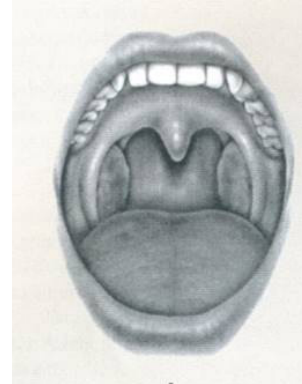

I

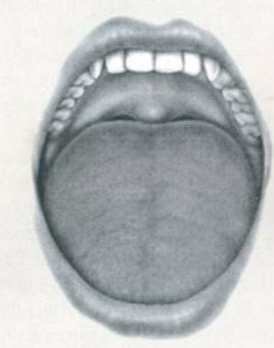

A

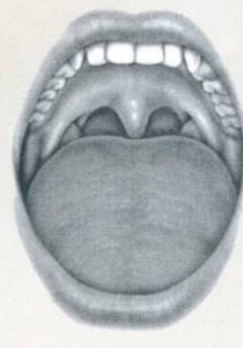

II

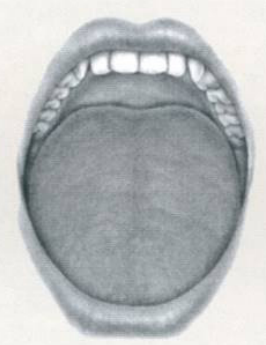

IV

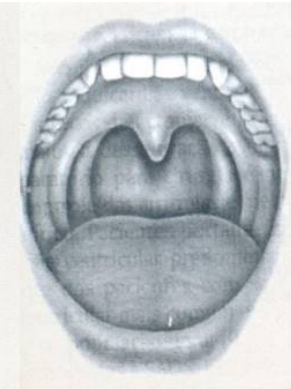

0

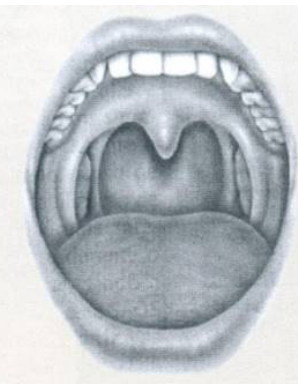

1

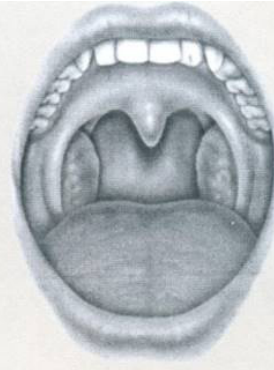

2

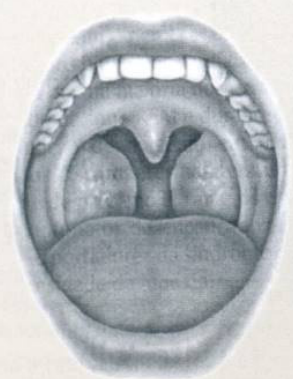

3

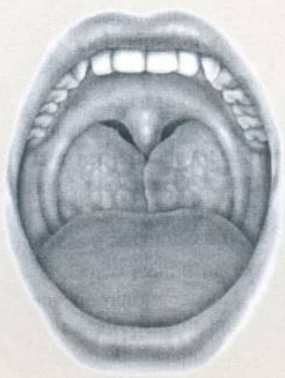

4

Figura 1. A) Escala de Mallampati (I a IV) que avalia a relação entre altura de base da língua e o grau de visualização das estruturas anatômicas na orofaringe. B) Classificação de Brodsky (0 a 4) para o grau de hipertrofia das tonsilas palatinas e sua projeção na linha média da orofaringe. 
redução leve no paladar e alguma dormência na língua. As raras complicações envolvem refluxo nasal permanente ou cicatrizes com uma retração levando a estenose palatal. ${ }^{25}$

Existem outras opções de procedimentos quando o sítio de obstrução está localizado no palato mole: a uvulopalatoplastia assistida por laser (LAUP), a radiofrequência em palato mole, os implantes palatais e a escleroterapia. São mais indicados em pacientes roncadores e com apneia leve em que o local de vibração e/ou obstrução seja especificamente o palato mole. Também podem ser realizados em associação a outros procedimentos cirúrgicos como parte do conceito de cirurgia em múltiplos níveis.

Outras técnicas cirúrgicas foram desenvolvidas mais recentemente para abordar casos em que se prevejam menores taxas de sucesso terapêutico após UPFP. Dentre elas, pode-se citar a faringoplastia lateral e a esfincterfaringoplastia expansiva. Pacientes que apresentam colapso faríngeo com tonsilas palatinas pequenas e Mallampati demonstrando obstrução na base da língua apresentam melhor taxa de sucesso após serem submetidos a tais variações técnicas.

A faringoplastia lateral é uma técnica mais funcional que a UPFP, atuando no espaço parafaríngeo e expandindo a VAS ao seccionar o músculo constrictor superior da faringe. O objetivo dessa cirurgia é mudar as propriedades constritoras da parede lateral da faringe, diferentemente do foco da UPFP, que é aumentar o lúmen da faringe pela remoção de tecidos. Ao compararem-se pacientes submetidos à UPFP e à faringoplastia lateral, um estudo observou melhores resultados clínicos e polissonográficos naqueles submetidos à faringoplastia lateral. ${ }^{26}$

\section{Cirurgias na hipofaringe}

A glossectomia da linha média (GLM) robótica e/ou a laser visa criar um alargamento de VAS retrolingual. As cirurgias que abordam a base de língua quando realizadas de forma isolada não levam a sucesso absoluto, já que obstrução das VAS na maioria das vezes ocorre em sítios múltiplos. Logo, sua eficácia é maior quando associada a outros procedimentos. ${ }^{27}$ As taxas de sucesso quando se associa UPFP com GLM variam de $76 \%$ a $100 \% .^{28-30}$

$\mathrm{O}$ avanço maxilomandibular (AMM) amplia as vias aéreas retrolingual e retropalatal, sendo a opção cirúrgica de maior sucesso no tratamento da SAOS. Essa técnica costuma ser mais realizada em quem não aceita CPAP nasal ou após outras cirurgias não terem sido bem-sucedidas. A mandíbula deve ser avançada de 8 a $14 \mathrm{~mm}$, com um sucesso cirúrgico que varia em torno de $75 \%$ a $100 \%{ }^{31-34}$ Esse procedimento geralmente é reservado aos pacientes graves nos quais os outros tratamentos falharam e/ou que não querem ser tratados com CPAP nasal. Pacientes submetidos a cirurgias anteriores podem fazer o AMM, que tem taxas de cura promissoras no longo prazo, mas o ganho de peso está associada com a recorrência da SAOS. ${ }^{35}$

\section{Cirurgia bariátrica}

A cirurgia bariátrica em pacientes com obesidade mórbida, quando consegue significativa perda de peso, melhora ou mesmo cura a SAOS. ${ }^{36,37}$ Trata-se de um método alternativo de manter a redução de peso a longo prazo que, além de melhorar a SAOS, garante um controle mais adequado da glicemia e reduz o risco de doenças. ${ }^{38}$ Uma revisão sistemática recente demonstrou que mais de 75\% dos pacientes submetidos à cirurgia bariátrica apresentaram melhora da SAOS. ${ }^{39}$

\section{Resultados cirúrgicos}

É difícil prever o resultado polissonográfico dos eventos obstrutivos nesses pacientes, principalmente nos mais graves, mas com uma indicação criteriosa espera-se haver uma melhora sintomática, ainda que incompleta. ${ }^{2}$ É sempre necessária uma polissonografia após cada procedimento cirúrgico, idealmente não antes de 4 meses após o procedimento, esperando uma redução no edema e reajuste dos reflexos respiratórios. Se há uso do CPAP nasal no pós-operatório, os pacientes devem suspender a terapia duas semanas antes do exame para permitir essa expressão de edema nas vias aéreas e uma potencial obstrução residual. Como a SAOS é uma condição muito associada ao envelhecimento, mesmo que haja um bom resultado cirúrgico inicial pode ocorrer retorno da síndrome ao longo dos próximos anos de vida e o CPAP sempre é uma possibilidade na terapia em pacientes de idade mais avançada. Esse fator deve sempre ser levado em conta pelo cirurgião que opera um paciente adulto jovem, na expectativa de se manterem as melhores condições anatômicas para uma boa adaptação ao CPAP nasal, como nariz pérvio e manutenção do mecanismo esfinctérico retropalatal.

\section{Conclusão}

O apelo por uma aparente intervenção curativa para a SAOS deve ser equilibrado com a incerteza em torno da sua segurança, eficácia continuada e impacto consistente em marcadores clínicos subjetivos e objeti- 


\section{Artigo de revisão}

vos. Há uma escassez nos dados de longo prazo quanto ao seguimento de pacientes tratados cirurgicamente, que nos poderiam ajudar a estabelecer sua resposta sintomática tardia, quais são as taxas de complicação e se aqueles optando por cirurgias requerem outras intervenções ao longo das décadas seguintes de vida. Embora a cirurgia possa não ser apropriada para todos os pacientes com SAOS, há subgrupos que podem se beneficiar muito e ensaios cirúrgicos randomizados e controlados devem continuar para esclarecermos essas questões. Mais pesquisas devem ser realizadas quanto à natureza da obstrução em VAS, visando formas de se identificarem os sítios de obstrução e uma padronização das técnicas cirúrgicas.

\section{Referências}

1. Guilleminault C, Stoohs R, Clerk A, et al. A cause of excessive daytime sleepiness: The upper airway resistance syndrome. Chest. 1993;104:781-7.

2. Yamadera W. Improvement in excessive daytime sleepiness after surgical treatment for obstructive sleep apnea syndrome. Psychiatry and Clinical Neurosciences. 1995;49(4):213-21.

3. Ferguson KA, Fleetham JA. Consequences of sleep disordered breathing. Thorax. 1995;50:998-1004.

4. American Sleep Disorders Association. Practice parameters for the treatment of obstructive sleep apnea in adults: the efficacy of surgical modifications of the upper airway. Sleep. 1996;19(2):152-5.

5. Kuhlo W, Doll E, Franck MC. Successful management of Pickwickian syndrome using long-term tracheostomy. Dtsch Med Wochenschr. 1969 Jun 13;94(24):1286-90.

6. Friedman M, Ibrahim H, Joseph NJ. Staging of obstructive sleep apnea/hypopnea syndrome: a guide to appropriate treatment. Laryngoscope. 2004;114: 454.

7. Sher AE, Schechtman KB, Piccirillo JF. The efficacy of surgical modifications of the upper airway in adults with obstructive sleep apnea syndrome [Review]. Sleep. 1996;19:156.

8. Carrol JL, Loughlin GM. Diagnostic criteria for obstructive sleep apnea syndrome in children. Pediatr Pulmonol. 1992;14:71-4.

9. Ansread M. Pediatric sleep disorders: new developments and evolving understanding. Curr Opin Pulm Med. 2000;6:501-6.

10. Bower C, Buckmiller L. What's new in pediatric sleep apnea? Curr Opin Otolaryngol Head Neck Surg. 2001;9:352-8.

11. Brunetti L, Rana S, Lospalluti ML, et al. Prevalence of obstructive sleep apnea in a cohort of 1207 children of Southern Italy. Chest. 2001;120:1930-5.

12. Schechter MS. Technical report: diagnosis and management of childhood obstructive sleep apnea. Pediatrics. 2002;109:69.

13. Nieminen P, Tolonen U, Lopponen $\mathrm{H}$. Snoring and obstructive sleep apnea in children - a 6-month follow-up study. Arch Otolaryngol Head Neck Surg. 2000;126:481-6.

14. Brietzke SE, Gallagher D. The effectiveness of tonsillectomy and adenoidectomy in the treatment of pediatric obstructive sleep apnea/hypopnea syndrome: A meta-analysis. Otolaryngology-Head and Neck Surgery. 2006;134:979-84.

15. American Academy of Pediatrics. Clinical practice guideline diagnosis and management of childhood obstructive sleep apnea syndrome. Pediatrics. 2002;109:704-12.
16. Lipton AJ, Gozal D. Treatment of obstructive sleep apnea in children. Do we really know how? Sleep Med Rev. 2003;7:61-80.

17. Darrow DH. Surgery for pediatric sleep apnea. Otolaryngol Clin North Am. 2007;40:855-75.

18. Rodenstein D, Collard P, Aubert G, et al. OSA treatment UPPP vs N-CPAP. Chest. 1995;107(2):584-5.

19. Hochban W, Brandenburg U, Peter JH. Surgical treatment of obstructive sleep apnea by maxilliary and mandibular osteotomy. Fortschritte der Kiefer - und Gesichts-Chirurgie. 1995;40:65-72.

20. Banerjee AR, Prichard AJ, Thomas RS. Surgery of the upper airway. Bailliere's Clinical Anaesthesiology. 1995;9(2):317-35.

21. Lin HC, Friedman M, Chang HW, et al. The efficacy of multilevel surgery of the upper airway in adults with obstructive sleep apnea/hypopnea syndrome. Laryngoscope. 2008;118:902-908.

22. Verse T, Baisch A, Maurer JT, et al. Multilevel surgery for obstructive sleep apnea: short-term results. Otolaryngol Head Neck Surg. 2006;134(4):571-77.

23. Hollandt JH, Mahlerwein M. Nasal breathing and continuous positive airway pressure (CPAP) in patients with obstructive sleep apnea (OSA). Sleep Breath. 2003 Jun;7(2):87-94.

24. Poirier J, George C, Rotenberg B. The effect of nasal surgery on nasal continuous positive airway pressure compliance. Laryngoscope. 2014 Jan;124(1):317-9.

25. Riley RW, Powell NP, Guilleminault C, et al. Obstructive sleep apnea surgery: risk, management and complications. Otolaryngol Head Neck Surg. 1997;177(6):648-52;

26. Cahali MB, Formigoni GG, Gebrim EM, et al. Lateral pharyngoplasty versus uvulopalatopharyngoplasty: a clinical, polysomnographic and computed tomography measurement comparison. Sleep. 2004;27(5):942-50.

27. Neruntarat C. Genioglossus advancement and hyoid myotomy: short-term and long term results. J Laryngol Otol. 2003;117(6):482-6.

28. Zhang Q, Zhang T, Li S, et al. Treatment result of uvulopalatopharyngoplasty and tongue base operation for the severe obstructive sleep apnea-hypopnea syndrome. (Chinese) Zhonghua Er Bi Yan Hou Ke Za Zhi. 2002;37(6):409-11.

29. Li HY, Wang PC, Hsu CY, et al. Same-stage palatopharyngeal and hypopharyngeal surgery for severe obstructive sleep apnea. ACTA Otolaryngol. 2004;124(7):820-6.

30. Suh GD. Evaluation of open midline glossectomy in the multilevel surgical management of obstructive sleep apnea syndrome. Otolaryngol Head Neck Surg. 2013;148(1):166-71.

31. Li KK, Riley RW, Powell NB, et al. Overview of phase II surgery for obstructive sleep apnea syndrome [Review]. Ear Nose Throat J. 1999;78:851-4.

32. Lee NR, Givens Jr CD, Wilson J, et al. Staged surgical treatment of obstructive sleep apnea syndrome: a review of 35 patients. J Oral Maxillofac Surg. 1999;57:382.

33. Bettega G, Pépin JL, Veale D, et al. Obstructive sleep apnea syndrome: fifty-one consecutive patients treated by maxillofacial surgery. Am J Respir Crit Care Med. 2000;162(2 Pt 1):641.

34. Riley NB, Powell RW, Guilleminault C. Maxillofacial surgery and obstructive sleep apnea: review of 80 patients. Otolaryngol Head Neck Surg. 1989;101:353.

35. Li KK, Powell NB, Riley RW, et al. Long-term results of maxillomandibular advancement surgery. Sleep Breath. 2000;4137.

36. Varela JE, Hinojosa MW, Nguyen NT. Resolution of obstructive sleep apnea after laparoscopic gastric bypass. Obes Surg. 2007;17:1279.

37. Fritscher LG, Canani S, Mottin CC, et al. Bariatric surgery in 
Lucas N. A. Lemes, Luciana B. Godoy. • Cirurgia no tratamento da síndrome da apneia obstrutiva do sono

the treatment of obstructive sleep apnea in morbidly obese patients. Respiration. 2007;74:647.

38. Ashrafian H, Darzi A, Athanasiou T. Bariatric surgery - can we afford to do it or deny doing it? Front Gastroenterol. 2011;2(2):82-9.

39. Sarkhosh K, Switzer NJ, El-Hadi M, et al. The impact of bariatric surgery on obstructive sleep apnea: a systematic review. Obes Surg. 2013;23(3):414-23. 